\title{
Urban Road Travel Time Prediction based on ELM
}

\author{
Lun $\mathrm{Li}^{1, \mathrm{a}}$, Dong Wang ${ }^{1, \mathrm{~b}}$, Zhu Xiao ${ }^{1, \mathrm{~b}}$, and Xiaohong $\mathrm{Li}^{1, \mathrm{~b}}$
}

\author{
${ }^{1}$ School of Information Science and Electronic Engineering, Hunan University, Changsha 410082 , \\ China; \\ alilunnu@126.com, ${ }^{\text {b }}$ wangd,zhxiao,lixhong\}@hnu.edu.cn
}

Keywords: Intelligent transportation systems(ITSs),TTP,ELM,prediction Model.

\begin{abstract}
The Travel Time Prediction (TTP) is an important element in the study of the advanced transportation guidance system and control system. In this paper, an advanced method with Extreme Learning Machine algorithm(ELM) has been discussed by analyzing the various travel time prediction method. The feasibility and advantages of Extreme Learning Machine in travel time prediction has been studied, and the comparisons between ELM and SVR and BPNN has been detaily discussed. Moreover, the traffic data, which is collected from REGIOLAB-DELFT platform, have been used for validation. The results show that the ELM algorithm outperforms the related value to those by SVR and BPNN.
\end{abstract}

\section{Introduction}

The Travel Time Prediction is valuable information required by drivers and transportation managers to improve the quality of travel and to make control decisions. Accuracy TTP enables drivers to make decisions, such as route choice and departure time. Besides, the TTP can be used by transportation agencies to deploy efficient control measures and to prevent potential traffic congestion[1]. The travel time and other variables(such as traffic speed,density,flow occupancy,etc) are directly used as the state variable in time series model methods or data-driven methods, and they can be used to predict travel times[2].Time series methods construct the time series relationship of travel time or traffic state, and then current and/or past traffic data are used in then constructed models to predict travel times in the next time interval[3]. The time series model include Kalman filter[4,5], auto-regressive integrated moving average model (ARIMA models)[5,6,7], data-driven methods predict travel time using large amount of historical traffic data,which include neural networks[8,9],support vector regression(SVR)[10,11].

However, the time series model assume that the travel time is linear trends and it does not reflect the actual road trip nonlinear characteristic. That leads to the low prediction reliability.The artificial intelligence method includes neural networks, support vector regression. The neural networks has the ability of self-organizing, self-learning, regardless of the internal structure of a mathematical model that can infinitely approximate and fit to nonlinear systems. Moreover, this network has the characteristics of adaptability and integration. However, the neural network is very difficult to overcome the shortcoming, such as complex network structure, over-fitting and some discrepancy between the predicted structure and expectations. For support vector machine regression, the training period is required to determine the parameters of the regression function, time-consuming. Compared to the traditional neural network, ELM has smaller training error and weights norm, faster speed for training and stronger generalization. Therefore, the ELM has been widely used in pattern recognition, time series prediction and so on.

The ELM has been used to predict the ultimate road travel time in this paper.we consider the impact of two previous link traffic flow and travel time for the next period link travel time Furthermore, the ELM prediction model has been established and its validate has been discussed by real-time data. 


\section{Methodology}

ELM algorithm. The ELM algorithm is a novel and fast learning method which was recently proposed by Huang et al[12]. The newly method belongs to Single-Layer Feedforward Neural Networks(SLFN). In the determining process of the network parameters, the optimal output weights can be determined analytically for a given training set under the following conditions.those are the activation function in the hidden layer can be infinitely differentiable,and the number of hidden layer neurons.Moreover, the least squares solutions can be obtained by minimizing quadratic loss function .As a result,the iteration is not needed when the network parameters is under determining leading to a great reducing of the amount of the computation and search place.

Algorithm theory. $\left(x_{i}, y_{i}\right)$ stands for a set of $\mathrm{N}$ arbitrary distinct samples, where $x_{i}=\left[x_{i 1}, x_{i 2}, \ldots, x_{i n}\right]^{T} \in R^{n}$ is the input vector, $y_{i}=\left[y_{i 1}, y_{i 2}, \ldots, y_{i n}\right]^{T} \in R^{n}$ is the output vector. A SLFN with $c$ hidden nodes and activation function $\left\{g\left(a_{i}, b_{i}, x\right)\right\}_{i=1}^{C}$ can be described as the following function.

$\sum_{i=1}^{c} \beta_{i} g_{i}\left(x_{j}\right)=\sum_{i=1}^{c} \beta_{i} g\left(a_{i} \cdot x_{j}+b_{i}\right)=y_{j}, j=1, \ldots, N$

where $a_{i}=\left[a_{i 1}, a_{i 2}, \ldots, a_{i m}\right]^{T}$ is the weight vector, which connects the ith hidden node and the input nodes; $b_{i}$ is the threshold of the ith hidden node; $\beta_{i}=\left[\beta_{i 1}, \beta_{i 2}, \ldots, \beta_{i n}\right]^{T}$ is the weight vector, and it is related to the ith hidden node and the output nodes. $g(x)$ is the activation function. The $\mathrm{N}$ samples satisfy the following equation

$$
H \beta=Y
$$

where $H$ is the hidden layer output matrix, and it can be defined as

$$
H\left(a_{1}, \ldots, a_{C}, b_{1}, \ldots, b_{C}, x_{1}, \ldots x_{N}\right)=\left[\begin{array}{ccc}
g\left(a_{1} \cdot x_{1}+b_{1}\right) & \mathrm{L} & g\left(a_{C} \cdot x_{1}+b_{C}\right) \\
\mathrm{M} & \mathrm{L} & \mathrm{M} \\
g\left(a_{1} \cdot x_{N}+b_{1}\right) & \mathrm{L} & g\left(a_{C} \cdot x_{N}+b_{C}\right)
\end{array}\right]_{N \times C}
$$

$$
\beta=\left[\begin{array}{c}
\beta_{1}^{T} \\
\vdots \\
\beta_{C}^{T}
\end{array}\right]_{C \times M} \quad \text { and } \quad Y=\left[\begin{array}{c}
y_{1}^{T} \\
\vdots \\
y_{N}^{T}
\end{array}\right]_{N \times M}
$$

Given hidden layer output matrix $H$ and the output $Y$, the output weights can be computed by Equation (2). The unique smallest norm least squares solution of the Equation(2) is:

$$
\hat{\beta}=H^{+} Y
$$

where $\mathrm{H}^{+}$is the Moore-Penrose which is generalized inverse of output matrix $H$.

The following is the construction of ELM algorithm:

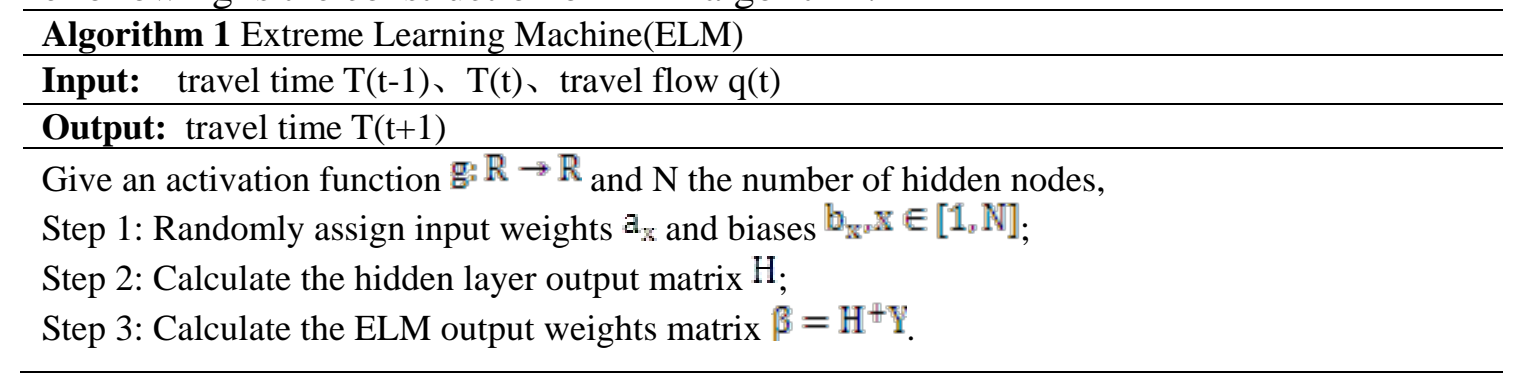

\section{The results and data analysis}

Experimental data sets. The object of this study comes from the city of Delft, the Netherlands Rijksweg urban road. One section of this road was predicted and analyzed. All the statistical data comes from the REGIOLAB-DELFT data platform[13]. Meanwhile the data derives from 00:00-24:00 on July 1, 2015 are selected to predict the traffic time, and the sampling rate is 3 minites. 
A total of 478 group traffic and travel time data are choosed, among which 400 groups of them are used to training and the remaining 78 groups data to validate the model. The predicted results can be obtained by the mapminmax of MATLAB after their prediction unnormalized.

Prediction Methodology and Error Measurements. Supposing that the current time is $t$, and we want to predict the future $y(t+1)$ at time $t+1$ with the knowledge of the value $y(t), y(t-1)$ for past time $\mathrm{t},(\mathrm{t}-1)$ and the current travel flow $\mathrm{q}(\mathrm{t})$. The prediction function can be expressed as

$$
y(t+1)=f(t, t-1, y(t), y(t-1), q(t)) \text {. }
$$

correlation coefficient $\mathrm{R}$ and root mean square error MSE are applied as performance indices:

$$
\begin{aligned}
& M S E=\frac{1}{N} \sum_{t=1}^{N}(R-\hat{R})^{2} \\
& R^{2}=1-\frac{\sum_{t=1}^{N}(R-\hat{R})^{2}}{\sum_{t=1}^{N}(R-\bar{R})^{2}}
\end{aligned}
$$

where $R$ is the actual value for the travel time, $\hat{R}$ is the travel-time prediction value, $\bar{R}$ is the average of the actual travel time, $\mathrm{N}$ is the number of samples of the simulation. MSE value obtained is small, the predicted value and the actual value getting more closer, the predicted effect is better than the compared algorithm.

Experimental Analysis. The travel time results based on three models can be plotted in Figure 1. Meanwhile prediction performance comparison is shown in Table 1.

Table 1 Three Algorithm comparing

\begin{tabular}{rlr}
\cline { 2 - 3 } Model & \multicolumn{1}{c}{ MSE } & \multicolumn{1}{c}{$\mathrm{R}^{2}$} \\
\hline ELM & 0.000074 & 0.0067 \\
SVR & 0.000807 & 0.0098 \\
BPNN & 0.0119 & 0.0019 \\
\hline
\end{tabular}
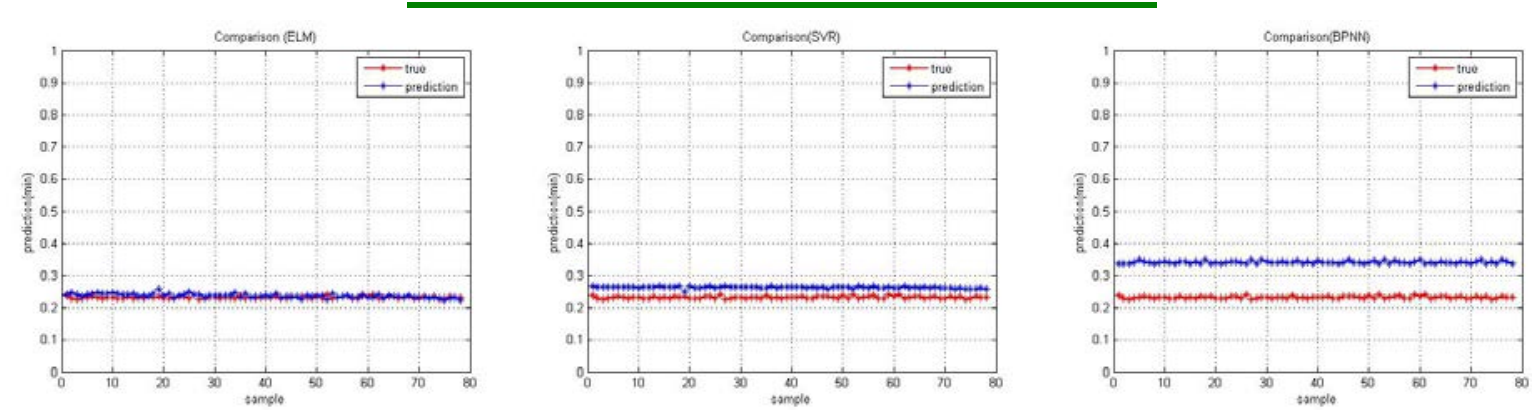

Fig.1 Three Algorithm Result

It can be seen from Figure 1 that ELM and SVR achieve a better result for travel time prediction than BPNN. In addition, From Table 1, the derived index, correlation coefficient and root mean square error of ELM are 0.0067 and 0.000074 , respectively. SVR correlation coefficient and root mean square error of 0.0098 and 0.000807 is similar, which is better than the BPNN. The optimal parameters select of SVR needs many iterations to complete,comsuming a lot of time. From the above analysis, the overall performance of ELM is better than SVR and BPNN

Experimental results show that ELM has obvious advantages on accuracy, parameters and learning speed compared with SVR and BPNN. Therefore,ELM has practical value in predicting travel time for travel time prediction 


\section{Summary}

This paper discussed the forecasting method based on Extreme Learning Machine. The urban road travel time prediction model has been established to predict future changes within a few short hours of travel time, and the model is verified by the real road data. Results show that, the ELM shows better result in travel time prediction compared to the SVR and BPNN algorithm. So, it can provide decision making for traffic guidance.

\section{Acknowledgment}

This work was supported in part by the National Natural Science Foundation of China(Grants Nos.61301148 and 61272061),the Fundamental Research Funds for the Central Universities of China(No.531107040263,531107040276),the research Funds for the Doctoral Program of Higher Education of China(Nos.20130161110002 and 20120161120019).

*Communication Author:Dong Wang(wangd@hnu.edu.cn)

\section{References}

[1] M. Yildirimoglu, N. Geroliminis,Experienced travel time prediction for congested freeways, J. Transportation Research Part B.53 (2013) 45-63.

[2] H.Chen, H.A.Rakha,C.C.McGhee,Dynamic Travel Time Prediction using Pattern Recognition, $20^{\text {th }}$ ITS Word Congress. Tokyo,2013.

[3] M.L.Yang,Y.G.Liu,Z.S.You,The Reliability of Travel Time Forecasting,J.IEEE Trans.Intell.Transport.Syst.,11(2010) 162-171.

[4] X.Fei,C.C.Lu,K.Liu,A Bayesian Dynamic Linear Model Approach for Real-time Short-term Freeway Travel Time Prediction,J.Transportation Research Part C:Emerging Technologies,19(2011) 1306-1318.

[5] J.S.Yang,Travel Time Prediction Using the GPS Test Vehicle and Kalman Filtering Techniques,in Proceedings of the 2005 American Control Conference,2005 2128-2133.

[6] J.X.Xia,M.Chen,W.Huang, A Multistep Corridor Travel-Time Prediction Method Using Presence-Type Vehicle Detector Data,J.Intelligent Transportation Systems:Technology,Planning,and Operations,15(2011)104-113.

[7] J.X.Xia,M.Chen,Dynamic Freeway Corridor Travel Time Prediction Using Single Inductive Loop Detector Data,Transportaion Research Board 88 ${ }^{\text {th }}$ Annual Meeting,2009.

[8] J.W.C.V.Lin,S.P.Hoogendoorn,H.J.V.Zuylen,Accurate Freeway Travel Time Prediction with State-space Neural Networks Under Missing Data,J.Transportation Research Part C 13(2005) 347-369.

[9] C.P.I.J.V.Hinsbergen,A.Hegyi,J.W.C.V.Lint,H.J.V.Zuylen,Bayesian Neural Networks for the Prediction of Stochastic Travel Times in Urban Networks,J.IET Intelligent Transport Systems,5(2011) 259-265.

[10] L.Vanajakshi,L.R.Rilett,Support Vector Machine Technique for the Short Term Prediction of Travel Time, in IEEE Intelligent Vehicles Symposium, Turkey, 2007.

[11] C.H.Wu, J.M.Ho, D. T. Lee, Travel-time Prediction with Support Vector Regression,J. IEEE Transactions on Intelligent Transportation Systems, 5(2004) 276-281 .

[12]H.G.Bin,Z.Q.Yu,C.K.Siew,Extreme learning machine: A new learning scheme of feedforward neural networks,J. IJCNN,2(2004) 985-990.

[13] Information on http://www.regiolab-delft.nl. 